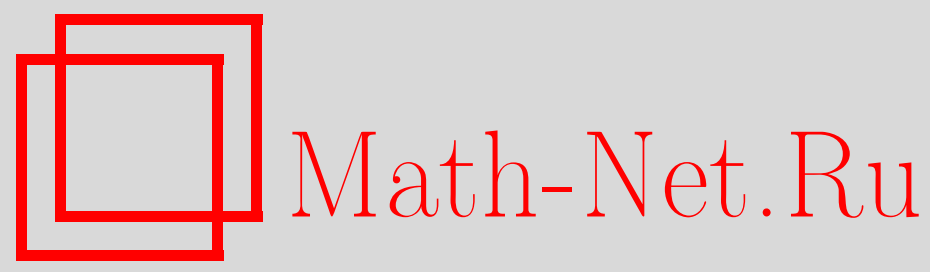

В. Г. Дурнев, Неразрешимость простого фрагмента позитивной теории с одной константой свободной полугруппы ранга 2, Матем. заметки, 2000, том 67, выпуск 2, 191-200

DOI: https://doi.org/10.4213/mzm827

Использование Общероссийского математического портала Math-Net.Ru подразумевает, что вы прочитали и согласны с пользовательским соглашением http://www.mathnet.ru/rus/agreement

Параметры загрузки:

IP: 54.198 .55 .26

26 апреля 2023 г., 13:04:32

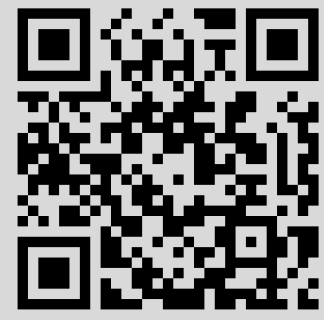




\title{
НЕРАЗРЕШИМОСТЬ ПРОСТОГО ФРАГМЕНТА ПОЗИТИВНОЙ ТЕОРИИ С ОДНОЙ КОНСТАНТОЙ СВОБОДНОЙ ПОЛУГРУППЫ РАНГА 2
}

\author{
В.Г. Дурнев
}

\begin{abstract}
В статье исследуется алгоритмическая природа некоторых "простых" фрагментов позитивных теорий с "малым" числом констант свободных нециклических полугрупп.

Библиография: 9 названий.
\end{abstract}

Через $\Pi_{n}^{-}$будем обозначать свободную полугруппу ранга $n$ без пустого слова со свободными образующими $a_{1}, \ldots, a_{n}$, а через $\Pi_{n}-$ свободную полугруппу с пустым словом ранга $n$ с теми же свободньпи образуюшими. Иногда $\Pi_{n}$ называют свободным моноидом ранга $n$.

Еще в 1946 году В. Куайн [1] доказал, что при $n \geqslant 2$ әлементарная теория свободной полугруппы $\Pi_{n}$, как и полугрупш $\Pi_{n}^{-}$, алгоритмически неразрешима.

С тех пор значительное внимание уделялось исследованию аналогичного вопроса для различных фрагментов элементарных теорий свободных полугрупп.

В работах [2], [3] для любого $n \geqslant 2$ построена формула

$$
P_{n}(x, y) \rightleftharpoons(\exists u)\left(\exists v_{1}\right)\left(\exists v_{2}\right)\left(\bigvee_{\substack{i, j=1 \\ i \neq j}}^{n}\left(x=u a_{i} v_{1} \& y=u a_{j} v_{2}\right) \vee \bigvee_{i=1}^{n}\left(x=y a_{i} v_{1} \vee y=x a_{i} v_{1}\right)\right)
$$

и доказано, что для любых двух элементов $g$ и $h$ полугрупшы $\Pi_{n}$ имеет место эквивалентность

$$
g \neq h \text { в полугрупе } \Pi_{n} \Longleftrightarrow \Pi_{n} \models P_{n}(g, h) .
$$

Нетрудно понять, как эту формулу преобразовать в аналогичную формулу для полугруппы $\Pi_{n}^{-}$.

Поэтому из указанного результата В. Куайна сразу следует алгоритмическая неразрешимость позитивных теорий полугрупn $\Pi_{n}$ и $\Pi_{n}^{-}$при $n \geqslant 2$, т.е. фрагментов элементарных теорий этих полугруп, состоящих из формул, в которые из пропозициональных связок входят лишь конъюнкция и дизъюнкция, а из теоремы Г. С. Маканина [4] о разрешимости проблемы совместности для систем уравнений в свободной полугруппе $\Pi_{n}\left(\Pi_{n}^{-}\right)$вытекает разрешимость экзистенииальной и универсальной теорий nолугрупn $\Pi_{n}$ и $\Pi_{n}^{-}$- фрагментов элементарных теорий этих полугруп, состоящих из

Работа выполнена при финансовой поддержке Российского фонда фундаментальных исследований. 
формул, имеющих предваренную нормальную форму с одним кванторньм блоком в приставке.

В 1973 году нами [5] доказана неразрешимость фрагмента әлементарной теории свободных полугрупп $\Pi_{n}$ u $\Pi_{n}^{-}$при $n \geqslant 2$, состоящего из позитивных формул $с$ кванторной приставкой типа $\exists \forall \exists^{3}$. В 1986 году С. С. Марченков в работе [6] доказал неразрешимость позитивной $\forall \exists^{4}$-теории каждой из зтих двух свободных полугрупп. Это улучшает результат работы [5] с точки зрения числа кванторных блоков в рассматриваемых формулах, но при этом общее число используемых кванторов в работах [5] и [6] одно и тоже. В 1995 году в работе [7] нами доказана алгоритмическая неразрешимость позитивной $\forall \exists^{3}$-теории каждой из двух свободных полугрупп $\Pi_{n}$ и $\Pi_{n}^{-}$при любом $n \geqslant 2$, что усиливает результаты работ [5] и [6].

Рассматриваемые в работах [6] и [7] формулы имеют достаточно простую кванторную приставку. В то же время их бескванторная часть содержит вхождения констант $a_{1}, \ldots, a_{n}$.

Естественно возникает вопрос о разрешимости “простых" фрагментов позитивных теорий с "мальм" числом констант полугрупп $\Pi_{n}$ и $\Pi_{n}^{-}$.

В 1986 году Н.А. Перязев в работе [8] доказал, что при $n \geqslant 2$ позитивная теория свободной полугруппы $\Pi_{n}$ с константами $a_{1}, \ldots, a_{n-2}$ разрешима, а с константами $a_{1}, \ldots, a_{n-1}$ алгоритмически неразрешима.

Целью настоящей заметки является установление алгоритмической природы некоторых “простых" фрагментов позитивных теорий с “мальп” числом констант полугрупा $\Pi_{n}$ и $\Pi_{n}^{-}$.

Следующая теорема существенно усиливает указанньй вьше результат Перязева об алгоритмической неразрешимости позитивной теории с константами $a_{1}, \ldots, a_{n-1}$ полугруппы $\Pi_{n}$.

ТЕоремА 1. При любом $n \geqslant 2$ позитивная $\forall^{3} \exists^{3}$-теория с константами $a_{1}, \ldots$, $a_{n-1}$ полугруппь $\Pi_{n}$ алгоритмически неразрешима, в то время как при любом $m$ позитивная $\forall \exists^{m}$-теория с константами $a_{1}, \ldots, a_{n-1}$ этой полугруппь разрешимa.

СлЕдСТВИЕ 1. Позитивная $\forall^{3} \exists^{3}$-теория с одной константой $a_{1}$ полугруппы $\Pi_{2}$ алгоритмически неразрешима, в то время как при любом $m$ позитивная $\forall^{1} \exists^{m}-$ теория с одной константой $a_{1}$ этой полугруппы разрешима.

Напомним, что в силу результата Перязева позитивная теория без констант полугруппь $\Pi_{2}$ разрешима.

ЗАмЕЧАнИЕ. Остается открытьм вопрос о разрешимости позитивных $\forall^{2} \exists^{m}$-теорий с константами $a_{1}, \ldots, a_{n-1}$ полугрупшы $\Pi_{n}$ при различных $m$, в частности, вопрос о разрешимости позитивных $\forall^{2} \exists^{m}$-теорий с одной константой $a_{1}$ полугруппы $\Pi_{2}$ при "небольших" $m$.

ТЕорема 2. При любом $n \geqslant 2$ илюбом $1 \leqslant k \leqslant n$ әлементарная $\forall^{k+1} \exists^{3}$-теория $c$ константами $a_{1}, \ldots, a_{n-k}$ полугруппы $\Pi_{n}$ алгоритмически неразрешима, в частности, алгоритмически неразрешима әлементарная $\forall^{n+1} \exists^{3}$-теория без констант этой полугруппь.

СлЕДСТВИЕ 2. Алгоритмически неразрешима әлементарная $\forall^{3} \exists^{3}$-теория без констант полугруппы $\Pi_{2}$. 
Выше уже отмечалось, что при любом $m$ элементарная $\forall^{m}$-теория и $\exists^{m}$-теория свободной полугруппы $\Pi_{2}$ разрешима [4].

ТЕорема 3. При любом $n \geqslant 2$ июбом $1 \leqslant k \leqslant n$ позитивная $\forall^{k+1} \exists^{3}$-теория с константами $a_{1}, \ldots, a_{n-k}$ полугруппы $\Pi_{n}^{-}$алгоритмически неразрешима, в то время как при любом $m$ позитивная $\forall^{k} \exists^{m}$-теория с константами $a_{1}, \ldots, a_{n-k}$ этой полугруппы разрешима.

СлЕДСТВИЕ 3. При любом $n \geqslant 2$ позитивная $\forall^{n+1} \exists^{3}-$ теория без констант полугруппь $\Pi_{n}^{-}$алгоритмически неразрешима, в то время как при любом $m$ позитивная $\forall^{n} \exists^{m}-$ теория без констант этой полугруппы разрешима.

СлЕДСТВИЕ 4. Позитивная $\forall^{3} \exists^{3}$-теория без констант полугруппы $\Pi_{2}^{-}$алгоритмически неразрешима, в то время как при любом m позитивная $\forall^{2} \exists^{m}$-теория без констант этой полугруппы алгоритмически разрешима.

ДоКАЗАТЕЛЬСТво ТЕОРЕМЫ 1. Как и в работе [6], в основу доказательства теоремы будет положено существование операторных алгоритмов с нерекурсивной областью определения [9], но в отличие от работы [6] мы воспользуемся операторными алгоритмами с "упрощенны.ми” командами.

Пусть $\mathfrak{A}$ - операторный алгоритм с нерекурсивной областью определения, программа которого состоит лишь из команд следуюшего вида:

$[\times 2]$ - умножить данное число на 2 и перейти $к$ выполнению следующей по порядку команды;

[×3] - умножить данное число на 3 и перейти к выполнению следующей по порядку команды;

[: $6 ; j]$ - если данное число делится на 6 , то разделить его на 6 и перейти к выполнению команды с номером $j$, в противном случае данное число не менять и перейти к выполнению следующей по порядку команды; [стоп] .

Предположим, что операторный алгоритм $\mathfrak{A}$ содержит $m$ команд, занумерованных числами от 1 до $m$, причем начальная команда имеет номер 1, а единственная команда [стоп] - номер $m$.

Напомним, что работа операторного алгоритма $\mathfrak{A}$ на входе $p$ начинается с выполнения команды с номером 1 ; в результате получаем число $p_{1}$ и номер следующей выполняемой команды $i_{1}$; по $p_{1}$ команда с номером $i_{1}$ дает число $p_{2}$ и номер следующей команды $i_{2}$ и т.д. Вычисление на входе $p$ будет закончено, когда на некотором шаге работы алгоритма в качестве номера следующей команды получится $m$ - номер команды [стоп].

Очевидно, что операторный алгоритм $\mathfrak{A}$ применим к числу р тогда и только тогда, когда существует последовательность

$$
\left(p_{0}, i_{0}\right),\left(p_{1}, i_{1}\right), \ldots,\left(p_{t}, i_{t}\right)
$$

такая, что $p_{0}=p, i_{0}=1, i_{t}=m$ и при любом $1 \leqslant j \leqslant t$ при применении $к p_{j-1}$ команды с номером $i_{j-1}$ получается число $p_{j}$ и номер следующей команды $i_{j}$.

Ради удобства будем использовать 0 и 1 вместо $a_{1}$ и $a_{2}$ соответственно.

В работе [6] последовательности (1) сопоставляется элемент полугрупшы $\Pi_{n}$

$$
0^{p_{0}+1} 1^{i_{0}} 0^{p_{1}+1} 1^{i_{1}} 0^{p_{2}+1} 1^{i_{2}} \ldots 0^{p_{t}+1} 1^{m},
$$


мы же сопоставим последовательности (1) следующий элемент этой полугруппы:

$$
0^{p_{0}+1} 1^{i_{0}+\varepsilon_{0} m} 0^{p_{1}+1} 1^{i_{1}+\varepsilon_{1} m} 0^{p_{2}+1} 1^{i_{2}+\varepsilon_{2} m} \ldots 0^{p_{t}+1} 1^{i_{t}+\varepsilon_{t} m}
$$

где $\varepsilon_{j} \rightleftharpoons 0$, если $p_{j}$ делится на 6, и 1 в противном случае $(j=0, \ldots, t)$.

Теперь, как это сделано в работах [6], [7], для произвольного натурального числа $p$ построим позитивную бескванторную формулу $\Phi_{p}\left(w, s, u, v, a_{1}, \ldots, a_{n}\right)$ такую, что на полугруппе $\Pi_{n}$ истинна формула

$$
(\forall w)(\exists s)(\exists u)(\exists v) \Phi_{p}\left(w, s, u, v, a_{1}, \ldots, a_{n}\right)
$$

тогда и только тогда, когда операторный алгоритм $\mathfrak{A}$ неприменим $к р$.

Для этого достаточно добиться, чтобы содержательно формула

$$
(\exists s)(\exists u)(\exists v) \Phi_{p}\left(w, s, u, v, a_{1}, \ldots, a_{n}\right)
$$

утверждала, что $w$ не имеет вид (2).

В качестве формулы $\Phi_{p}\left(w, s, u, v, a_{1}, \ldots, a_{n}\right)$ можно взять дизъюнкцию следующих формул 1)-9).

1) Слово $w$ пусто: $w w=w$.

2) Слово $w$ содержит вхождение некоторой буквы $a_{i}$ при $3 \leqslant i \leqslant n$ :

$$
\bigvee_{i=3}^{n} w=u a_{i} v
$$

3) Слово $w$ не начинается ни с $0^{p+1} 10$, ни с $0^{p+1} 1^{1+m} 0$ :

$$
\begin{aligned}
& \left(\bigvee_{\substack{i, j=1 \\
i \neq j}}^{n}\left(0^{p+1} 10=u a_{i} s \& w=u a_{j} v\right) \vee \bigvee_{i=1}^{n} 0^{p+1} 10=w a_{i} u\right) \\
& \quad \&\left(\bigvee_{\substack{i, j=1 \\
i \neq j}}^{n}\left(0^{p+1} 1^{1+m} 0=u a_{i} s \& w=u a_{j} v\right) \vee \bigvee_{i=1}^{n} 0^{p+1} 1^{1+m} 0=w a_{i} u\right)
\end{aligned}
$$

4) Слово $w$ не оканчивается ни на $01^{m}$, ни на $01^{m+m}$ :

$$
w 1=1 w \vee \bigvee_{0 \leqslant i<m} w=u 01^{i} \vee \bigvee_{1 \leqslant i<m} w=u 01^{i+m} \vee w=u 1^{2 m+1}
$$

5) Слово $w$ содержит в качестве подслова слово $1^{2 m+1}: w=u 1^{2 m+1} v$.

6) Слово $w$ содержит $1^{m}$ или $1^{2 m}$ не в конще: $w=u 01^{m} 0 v \vee w=u 01^{2 m} 0 v$.

Заметим, что если для слова $w$ не вьполняется ни одно из условий 1$)-6)$, то $w$ имеет вид

$$
0^{p+1} 1^{i_{0}} 0^{p_{1}+1} 1^{i_{1}} 0^{p_{2}+1} 1^{i_{2}} \ldots 0^{p_{t}+1} 1^{i_{t}}
$$

для некоторых целых неотрищательньх чисел $p_{1}, \ldots, p_{t}$ и натуральных чисел $i_{0}, i_{1}, i_{2}$, $\ldots, i_{t}$, причем $i_{0}$ - это 1 или $1+m, i_{t}$ - это $m$ или $2 m, 1 \leqslant i_{j} \leqslant 2 m(1 \leqslant j \leqslant t)$, причем при $j<t i_{j}$ отлично и от $m$, и от $2 m$. 
7) Напомним, что если $p_{j}$ делится на 6 , то $1 \leqslant i_{j} \leqslant m$; в противном случае имеем $m+1 \leqslant i_{j} \leqslant 2 m$, это условие нарушается с помощью следующей формулы:

$$
\begin{aligned}
s 0= & 0 s \&\left(w=0 s^{6} 1^{1+m} u \vee \bigvee_{1 \leqslant \varepsilon \leqslant 5} w=00^{\varepsilon} s^{6} 10 u\right. \\
& \vee w=u 10 s^{6} 1^{m+m} \vee \bigvee_{1 \leqslant \varepsilon \leqslant 5} w=u 100^{\varepsilon} s^{6} 1^{m} \\
& \left.\vee \bigvee_{1 \leqslant i \leqslant m} w=u 10 s^{6} 1^{i+m} 0 v \vee \bigvee_{1 \leqslant \varepsilon \leqslant 5} \bigvee_{1 \leqslant i \leqslant m} w=u 100^{\varepsilon} s^{6} 1^{i} 0 v\right) .
\end{aligned}
$$

8) Каждому числу $i$, являющемуся номером команды вида $[\times d]$, где $d=2,3$, сопоставим формулу, которая содержательно утверждает, что в слове $w$ в некотором месте произошел “сбой” из-за неправильного выполнения әтой командь, т.е. либо неправильно вычислен результат выполнения этой команды, либо не то число указано в качестве номера следующей команды.

В качестве такой формулы возьмем формулу вида

$$
s 0=0 s \&\left(\Psi_{1} \vee \Psi_{2}\right),
$$

где формула $\Psi_{1}$ будет содержательно утверждать, что неправильно вычислен результат выполнения команды вида $[\times d]$, а формула $\Psi_{2}$ содержстельно будет утверэдать, что результат применения команды $[\times d]$ вычислен правильно, но неправильно указан номер следующей команды (при условии, что $s$-степень 0).

Пусть $\Psi_{1}-$ следующая формула:

$$
\begin{gathered}
\left(\bigvee_{\varepsilon=0,1} w=0 s 1^{i+\varepsilon m} 0 s^{d} 0 u\right) \vee\left(\bigvee_{\varepsilon=0,1} \bigvee_{1 \leqslant l \leqslant d-1} w=v 01^{i+\varepsilon m} 0 s^{d} 0^{l} 1 u\right) \\
\quad \vee\left(\bigvee_{\varepsilon=0,1} w=v 00 s 1^{i+\varepsilon m} 0 s^{d} 1 u\right) \vee\left(\bigvee_{\varepsilon=0,1} w=v 1 s 01^{i+\varepsilon m} 0 s^{d} 0 u\right) .
\end{gathered}
$$

А в качестве $\Psi_{2}$ возьмем формулу

$$
\begin{aligned}
& \bigvee_{\varepsilon=0,1} \bigvee_{l=0,1} \bigvee_{\substack{j=1 \\
j \neq i+1}}^{m}\left(w=0 s 1^{i+\varepsilon m} 0 s^{d} 1^{j+l m} 0 u\right. \\
& \left.\vee w=v 10 s 1^{i+\varepsilon m} 0 s^{d} 1^{j+l m} 0 u \vee w=v 10 s 1^{i+\varepsilon m} 0 s^{d} 1^{j+l m} \vee w=0 s 1^{i+\varepsilon m} 0 s^{d} 1^{j+l m}\right) .
\end{aligned}
$$

9) Каждому $i$, являющемуся номером команды вида [: $6 ; j]$, сопоставим формулу, которая содержательно утверждает, что в слове $w$ в некотором месте произошел 
“сбой” из-за неправильного выполнения этой команды, т.е. либо неправильно вычислен результат выполнения әтой команды, либо не то число указано в качестве номера следующей команды:

$$
\begin{aligned}
& s 0=0 s \&\left(w=0 s^{6} 1^{i} 0 s 0 u \vee w=v 00 s^{6} 1^{i} 0 s 1 u\right. \\
& \vee w=v 10 s^{6} 1^{i} 0 s 0 u \\
& \vee \bigvee \bigvee_{\varepsilon=0,1} \bigvee_{\substack{t \neq j \\
t \neq j}}^{m}\left(w=0 s^{6} 1^{i} 0 s 1^{t+\varepsilon m} 0 u \vee w=0 s^{6} 1^{i} 0 s 1^{t+\varepsilon m}\right. \\
& \left.\vee w=v 10 s^{6} 1^{i} 0 s 1^{t+\varepsilon m} 0 u \vee w=v 10 s^{6} 1^{i} 0 s 1^{t+\varepsilon m}\right) \\
& \vee w=s 01^{i+m} s 00 u \vee w=v 00 s 1^{i+m} 0 s 1 u \\
& \vee w=v 1 s 01^{i+m} s 00 u \\
& \vee \bigvee \bigvee_{\varepsilon=0,1}^{\substack{t=1 \\
t \neq i+1}}\left(w=0 s 1^{i+m} 0 s 1^{t+\varepsilon m} 0 u \vee w=0 s 1^{i+m} 0 s 1^{t+\varepsilon m}\right. \\
& \left.\left.\vee w=v 10 s 1^{i+m} 0 s 1^{t+\varepsilon m} 0 u \vee w=v 10 s 1^{i+m} 0 s 1^{t+\varepsilon m}\right)\right) .
\end{aligned}
$$

Сделаем некоторые пояснения к последней формуле: первые четыре строки относятся к случаю, когда число, к которому применяется $i$-я команда $[: 6 ; j]$, делится на 6 , причем, в первых двух строках утверждается, что неправильно вычислен результат, т.е. он не получается из предыдущего числа делением на 6, а следующие две строки утверждают, что результат вычислен правильно, но неправильно указан номер следующей команды. Аналогично четыре последние строки относятся к случаю, когда число, к которому применяется $i$-я команда [: $6 ; j]$, не делится на 6 , причем, первые две строки последней четверки утверждают, что неправильно вычислен результат (т.е. число, к которому применяется команда, изменено, хотя в данном случае оно не должно менятся), а две последние строки утверждают, что результат вычислен правильно, но неправильно указан номер следующей команды.

Дизъюнкцию построенных вьше формул 1)-7) и формул вида 8), 9), отвечаюших значениям $i$ номеров соответствующих команд, обозначим через $\Phi_{p}\left(w, s, u, v, a_{1}, \ldots, a_{n}\right)$.

Тогда для произвольного натурального числа $p$ имеет место эквивалентность: на полугруппе $\Pi_{n}$ истинна формула

$$
(\forall w)(\exists s)(\exists u)(\exists v) \Phi_{p}\left(w, s, u, v, a_{1}, \ldots, a_{n}\right)
$$

тогда и только тогда, когда операторный алгоритм $\mathfrak{A}$ неприменим к $р$.

Построим формулу $\Psi_{p}$, полагая

$$
\begin{aligned}
\Psi_{p} & \rightleftharpoons(\forall t)(\forall q)(\forall w)(\exists s)(\exists u)(\exists v)(t t=t \vee q q=q \\
& \vee \bigvee_{i=1}^{n-1}\left(t=u a_{i} v \vee q=u a_{i} v\right) \vee\left(t=u s \& q s=v^{3}\right) \\
& \left.\vee \Phi_{p}\left(w, s, u, v, a_{1}, \ldots, a_{n-1}, t\right)\right)
\end{aligned}
$$


Покажем, что имеет место эквивалентность

$$
\Pi_{n} \vDash(\forall w)(\exists s)(\exists u)(\exists v) \Phi_{p}\left(w, s, u, v, a_{1}, \ldots, a_{n-1}, a_{n}\right) \Longleftrightarrow \Pi_{n}=\Psi_{p}
$$

Обозначим через $\mathscr{A}(t, q)$ формулу

$$
(\exists s)(\exists u)(\exists v)\left(t t=t \vee q q=q \vee \bigvee_{i=1}^{n-1}\left(t=u a_{i} v \vee q=u a_{i} v\right) \vee\left(t=u s \& q s=v^{3}\right)\right)
$$

а через $\mathscr{B}(t)$ формулу

$$
(\forall w)(\exists s)(\exists u)(\exists v) \Phi_{p}\left(w, s, u, v, a_{1}, \ldots, a_{n-1}, t\right) .
$$

Тогда формула $\Psi_{p}$ равносильна формуле $(\forall t)((\forall q) \mathscr{A}(t, q) \vee \mathscr{B}(t))$.

Если формула $\Psi_{p}$ истинна на полугруппе $\Pi_{n}$, то истинна, в частности, и формула

$$
\begin{aligned}
(\exists s) & (\exists u)(\exists v)\left(a_{n} a_{n}=a_{n} \vee a_{n} a_{n}=a_{n}\right. \\
& \left.\vee \bigvee_{i=1}^{n-1}\left(a_{n}=u a_{i} v \vee a_{n}=u a_{i} v\right) \vee\left(a_{n}=u s \& a_{n} s=v^{3}\right)\right) \\
& \vee(\forall w)(\exists s)(\exists u)(\exists v) \Phi_{p}\left(w, s, u, v, a_{1}, \ldots, a_{n-1}, a_{n}\right) .
\end{aligned}
$$

Но, очевидно, формула

$$
\begin{gathered}
(\exists s)(\exists u)(\exists v)\left(a_{n} a_{n}=a_{n} \vee a_{n} a_{n}=a_{n}\right. \\
\left.\vee \bigvee_{i=1}^{n-1}\left(a_{n}=u a_{i} v \vee a_{n}=u a_{i} v\right) \vee\left(a_{n}=u s \& a_{n} s=v^{3}\right)\right)
\end{gathered}
$$

ложна на полугруппе $\Pi_{n}$, поэтому истинна формула

$$
(\forall w)(\exists s)(\exists u)(\exists v) \Phi_{p}\left(w, s, u, v, a_{1}, \ldots, a_{n-1}, a_{n}\right) .
$$

Чтобы доказать обратное утверждение, предположим, что на полугруппе $\Pi_{n}$ истинна формула

$$
(\forall w)(\exists s)(\exists u)(\exists v) \Phi_{p}\left(w, s, u, v, a_{1}, \ldots, a_{n-1}, a_{n}\right)
$$

и покажем, что тогда истинна и формула $\Psi_{p}$.

Пусть $t_{0}, q_{0}$ - произвольные элементы полугруппы $\Pi_{n}$.

Покажем, что истинна формула

$$
\begin{aligned}
& (\forall w)(\exists s)(\exists u)(\exists v)\left(t_{0} t_{0}=t_{0} \vee q_{0} q_{0}=q_{0}\right. \\
& \quad \vee \bigvee_{i=1}^{n-1}\left(t_{0}=u a_{i} v \vee q_{0}=u a_{i} v\right) \vee\left(t_{0}=u s \& q_{0} s=v^{3}\right) \\
& \left.\vee \Phi_{p}\left(w, s, u, v, a_{1}, \ldots, a_{n-1}, t_{0}\right)\right)
\end{aligned}
$$


Но последняя формула равносильна формуле $\mathscr{A}\left(t_{0}, q_{0}\right) \vee \mathscr{B}\left(t_{0}\right)$.

Если $t_{0}$ или $q_{0}$ не является ненулевой степенью буквы $a_{n}$, то истинна формула

$$
(\exists s)(\exists u)(\exists v)\left(t_{0} t_{0}=t_{0} \vee q_{0} q_{0}=q_{0} \vee \bigvee_{i=1}^{n-1}\left(t_{0}=u a_{i} v \vee q_{0}=u a_{i} v\right)\right),
$$

а значит, и формулы $\mathscr{A}\left(t_{0}, q_{0}\right)$ и $\mathscr{A}\left(t_{0}, q_{0}\right) \vee \mathscr{B}\left(t_{0}\right)$.

Пусть $t_{0}$ и $q_{0}$ - ненулевые степени буквы $a_{n}$.

Если $t_{0}=a_{n}^{r}$ и $r \geqslant 2$, то истинна формула

$$
(\exists s)(\exists u)(\exists v)\left(t_{0}=u s \& q_{0} s=v^{3}\right),
$$

а значит, и формулы $\mathscr{A}\left(t_{0}, q_{0}\right)$ и $\mathscr{A}\left(t_{0}, q_{0}\right) \vee \mathscr{B}\left(t_{0}\right)$.

Если же $t_{0}=a_{n}$, то истинна формула

$$
(\forall w)(\exists s)(\exists u)(\exists v) \Phi_{p}\left(w, s, u, v, a_{1}, \ldots, a_{n-1}, t_{0}\right),
$$

а значит, и формула $\mathscr{A}\left(t_{0}, q_{0}\right) \vee \mathscr{B}\left(t_{0}\right)$.

Из истинности формулы $\mathscr{A}\left(t_{0}, q_{0}\right) \vee \mathscr{B}\left(t_{0}\right)$ следует истинность и равносильной ей формулы

$$
\begin{aligned}
& (\forall w)(\exists s)(\exists u)(\exists v)\left(t_{0} t_{0}=t_{0} \vee q_{0} q_{0}=q_{0}\right. \\
& \quad \vee \bigvee_{i=1}^{n-1}\left(t_{0}=u a_{i} v \vee q_{0}=u a_{i} v\right) \vee\left(t_{0}=u s \& q_{0} s=v^{3}\right) \\
& \left.\quad \vee \Phi_{p}\left(w, s, u, v, a_{1}, \ldots, a_{n-1}, t_{0}\right)\right) .
\end{aligned}
$$

Так как $t_{0}, q_{0}$-произвольные элементы полугрупш $\Pi_{n}$, получаем, что на полугрупе $\Pi_{n}$ истинна формула $\Psi_{p}$.

Поэтому для произвольного натурального числа $p$ имеет место эквивалентность: на полугруппе $\Pi_{n}$ истинна формула $\Psi_{p}$ тогда и только тогда, когда операторный алгоритм А неприменим к р.

А так как по предположению операторньй алгоритм $\mathfrak{A}$ имеет нерекурсивную область определения, тем самым доказано, что при любом $n \geqslant 2$ позитивная $\forall^{3} \exists^{3}$-теория с константами $a_{1}, \ldots, a_{n-1}$ полугруппы $\Pi_{n}$ алгоритмически неразрешима.

Покажем, что для любого натурального числа $m$ алгоритмически разрешима позитивная $\forall \exists^{m}$-теория с константами $a_{1}, \ldots, a_{n-1}$.

Пусть $F$ - позитивная $\forall \exists^{m}$-формула, бескванторная часть которой содержит лишь константы $a_{1}, \ldots, a_{n-1}$.

Можно считать, что $F$ имеет вид

$$
\left(\forall x_{1}\right)\left(\exists y_{1}\right) \ldots\left(\exists y_{m}\right) \Psi\left(x_{1}, y_{1}, \ldots, y_{m}, a_{1}, \ldots, a_{n-1}\right),
$$

где $\Psi\left(x_{1}, y_{1}, \ldots, y_{m}, a_{1}, \ldots, a_{n-1}\right)$ - бескванторная часть.

Нетрудно понять, что имеет место следующая эквивалентность:

$$
\begin{aligned}
\Pi_{n} & =\left(\forall x_{1}\right)\left(\exists y_{1}\right) \ldots\left(\exists y_{m}\right) \Psi\left(x_{1}, y_{1}, \ldots, y_{m}, a_{1}, \ldots, a_{n-1}\right) \\
& \Longleftrightarrow \Pi_{n} \mid=\left(\exists y_{1}\right) \ldots\left(\exists y_{m}\right) \Psi\left(a_{n}, y_{1}, \ldots, y_{m}, a_{1}, \ldots, a_{n-1}\right) .
\end{aligned}
$$

Для завершения доказательства теоремы 1 остается воспользоваться результатом Маканина [4] об алгоритмической разрешимости проблемы совместности для систем уравнений в свободных полугрупшах. 
ДоКАЗАТЕЛЬСТВо ТЕОРЕМЫ 2 . По формуле $\Phi_{p}\left(w, s, u, v, a_{1}, \ldots, a_{n}\right)$ построим формулу $F_{k, p}$, полагая

$$
\begin{aligned}
F_{k, p} & \rightleftharpoons\left(\forall y_{1}\right) \ldots\left(\forall y_{k}\right)(\forall w)(\exists s)(\exists u)(\exists v)\left(\bigvee_{1 \leqslant i<j \leqslant k} y_{i}=y_{j}\right. \\
& \vee \bigvee_{i=1}^{k}\left(y_{i}=y_{i} y_{i} \vee\left(y_{i}=u v \& u \neq u u \& v \neq v v\right)\right) \\
& \left.\vee \bigvee_{i=1}^{k} \bigvee_{j=1}^{n-k} y_{i}=a_{j} \vee \Phi_{p}\left(w, s, u, v, a_{1}, \ldots, a_{n-k}, y_{1}, \ldots, y_{k}\right)\right)
\end{aligned}
$$

Нетрудно понять, что имеет место эквивалентность

$$
\Pi_{n} \vDash(\forall w)(\exists s)(\exists u)(\exists v) \Phi_{p}\left(w, s, u, v, a_{1}, \ldots, a_{n}\right) \Longleftrightarrow \Pi_{n} \models F_{k, p} .
$$

Это завершает доказательство теоремы 2.

ДоКАЗАТЕЛЬСТВо ТЕОРЕМЫ 3. Подобно тому, как это сделано в ходе доказательства теоремы 1, по операторному алгоритму $\mathfrak{A}$ с нерекурсивной областью определения и по натуральному числу $p$ построим позитивную бескванторную формулу $\Phi_{p}(w, s, u, v$, $\left.a_{1}, \ldots, a_{n}\right)$ такую, что для произвольного натурального числа $p$ имеет место эквивалентность: на полугруппе $\Pi_{n}^{-}$истинна формула

$$
(\forall w)(\exists s)(\exists u)(\exists v) \Phi_{p}\left(w, s, u, v, a_{1}, \ldots, a_{n}\right)
$$

тогда и только тогда, когда операторный алгоритм $\mathfrak{A}$ неприменим к $р$.

Для этого потребуется внести очевидные, хотя и достаточно громоздкие, изменения в ход построения в процессе доказательства теоремы 1 формулы $\Phi_{p}\left(w, s, u, v, a_{1}, \ldots, a_{n}\right)$, вызванные отсутствием в полугруппе $\Pi_{n}^{-}$пустого слова.

Построим формулу $F_{k, p}$, полагая

$$
\begin{gathered}
F_{k, p} \rightleftharpoons\left(\forall y_{1}\right) \ldots\left(\forall y_{k}\right)(\forall w)(\exists s)(\exists u)(\exists v)\left(\bigvee_{1 \leqslant i<j \leqslant k} y_{i}=y_{j} \vee \bigvee_{i=1}^{n} y_{i}=u v\right. \\
\left.\vee \bigvee_{i=1}^{k} \bigvee_{j=1}^{n-k} y_{i}=a_{j} \vee \Phi_{p}\left(w, s, u, v, a_{1}, \ldots, a_{n-k}, y_{1}, \ldots, y_{k}\right)\right) .
\end{gathered}
$$

Нетрудно понять, что для произвольного натурального числа $p$ имеет место эквивалентность

$$
\Pi_{n}^{-} \models(\forall w)(\exists u)(\exists v)(\exists s) \Phi_{p}\left(w, s, u, v, a_{1}, \ldots, a_{n}\right) \Longleftrightarrow \Pi_{n}^{-} \vDash F_{k, p} .
$$

Поэтому на полугруппе $\Pi_{n}^{-}$истинна формула $F_{k, p}$ тогда и только тогда, когда операторный алгоритм $\mathfrak{A}$ неприменим $\kappa р$.

А так как по предположению операторньй алгоритм $\mathfrak{A}$ имеет нерекурсивную область определения, тем самьм доказано, что при любом $n \geqslant 2$ и любом $1 \leqslant k \leqslant n$ позитивная $\forall^{k+1} \exists^{3}-$ теория с константами $a_{1}, \ldots, a_{n-k}$ полугруппы $\Pi_{n}^{-}$алгоритмически неразрешима. 
Докажем, что для любого натурального числа $m$ алгоритмически разрешима позитивная $\forall^{k} \exists^{m}$-теория с константами $a_{1}, \ldots, a_{n-k}$.

Пусть $\Phi$ - позитивная $\forall^{k} \exists^{m}$-формула, бескванторная часть которой содержит лишш константы $a_{1}, \ldots, a_{n-k}$.

Можно считать, что $\Phi$ имеет вид

$$
\left(\forall x_{1}\right) \ldots\left(\forall x_{k}\right)\left(\exists y_{1}\right) \ldots\left(\exists y_{m}\right) \Psi\left(x_{1}, \ldots, x_{k}, y_{1}, \ldots, y_{m}, a_{1}, \ldots, a_{n-k}\right)
$$

где $\Psi\left(x_{1}, \ldots, x_{k}, y_{1}, \ldots, y_{m}, a_{1}, \ldots, a_{n-k}\right)$ - бескванторная часть.

Нетрудно понять, что имеет место следующая эквивалентность:

$$
\begin{aligned}
\Pi_{n}^{-} & =\left(\forall x_{1}\right) \ldots\left(\forall x_{k}\right)\left(\exists y_{1}\right) \ldots\left(\exists y_{m}\right) \Psi\left(x_{1}, \ldots, x_{k}, y_{1}, \ldots, y_{m}, a_{1}, \ldots, a_{n-k}\right), \\
& \Longleftrightarrow \Pi_{n}^{-} \models\left(\exists y_{1}\right) \ldots\left(\exists y_{m}\right) \Psi\left(a_{n-k+1}, \ldots, a_{n}, y_{1}, \ldots, y_{m}, a_{1}, \ldots, a_{n-k}\right) .
\end{aligned}
$$

Для завершения доказательства теоремы 3 остается, как и вьше, воспользоваться результатом Маканина [4].

Выражаю глубокую благодарность чл.-корр. РАН С. И. Адяну за внимание к работе и полезные замечания, высказанные им при обсуждении излагаемых в работе результатов.

\section{СПИСОК ЦИТИРОВАННОЙ ЛИТЕРАТУРЫ}

[1] Quine W. Concatenation as a basis for arithmetic // J. Symbolic Logic. 1946. V. 11. P. 105-114.

[2] Дурнев В.Г. О позитивной теории свободной полугруппы // Вопросы теории групп и полугрупп. Тула, 1972. С. 122-172.

[3] Косовский Н. К. Элементы математической логики и ее приложения к теории субрекурсивных алгоритмов. Л.: Изд-во ЛГУ, 1981.

[4] Маканин Г. С. Проблема разрешимости уравнений в свободной полугруппе // Матем. сб. 1977. T. 103 (145). № $2(6)$. С. 147-236.

[5] Дурнев В.Г. Позитивная теория свободной полугруппы // Докл. АН СССР. 1973. T. 211. № 4. С. $772-774$.

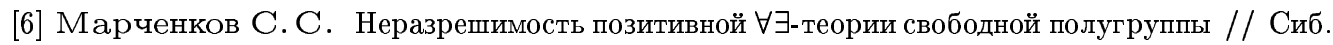
матем. ж. 1982. Т. 23. №1. С. 196-198.

[7] Дурнев В. Г. Неразрешимость позитивной $\forall \exists^{3}$-теории свободной полугруппы // Сиб. матем. ж. 1995. Т. 36. № 5. С. 1067-1080.

[8] Перязев Н. А. О позитивной эквивалентности свободных алгебр // Восьмая Всесоюзная конференция по математической логике. Тезисы докл. М., 1986. С. 149.

[9] Мальцев А. И. Алгоритмы и рекурсивные функции. М.: Наука, 1965.

Ярославский государственный университет им. П. Г. Демидова

E-mail: durnev@univ.uniyar.ac.ru

Поступило

27.04.1998

Исправленный вариант

08.09.1999 